\title{
Bunch Shape Monitor For SSCL Linac*
}

\author{
J. W. Hurd, G. M. Arbique, C. E. Crist, F. W. Guy, G. T. Leifeste, D. Raparia, K. Saadatmand, D. A. Swenson \\ Superconducting Super Collider Laboratory \\ 2550 Beckleymeade Avenue, Dallas, TX 75237
}

\author{
S. Esin, A. Feschenko, A. Stepanov, A. Mirzojan \\ Institute for Nuclear Research of the Academy of Sciences of Russia \\ Moscow, 117312
}

\section{Abstract}

The Superconducting Super Collider Laboratory and the Institute for Nuclear Research are collaboratively developing a Bunch Shape Monitor [1] diagnostics for commission the SSCL linac. The Bunch Shape Monitor is designed to measure the intensity of beam as a function of time over the micro-bunch of the beam. Design resolution for the SSCL monitors is approximately 7 psec. The first monitor will operate at the fundamental frequency of $428 \mathrm{MHz}$ and will be used to measure the output beam of the RFQ Linac. First available results will be presented and compared with predictions. Further development will allow the monitors to fit in a standard SSCL beam box and one will operate at the third harmonic of $428 \mathrm{MHz}$. Proposals to use the Bunch Shape Monitor to measure the longitudinal phase space distribution of the beam will be discussed.

\section{INTRODUCTION}

The SSCL linac [2] consists of three major if accelerators, a Radio Frequency Quadrupole that accelerates an $\mathrm{H}^{-}$beam to $2.5 \mathrm{MeV}$, a Drift Tube Linac to $70 \mathrm{MeV}$, and a Coupled Cavity Linac to $600 \mathrm{MeV}$. The $\mathrm{H}^{-}$beam is then stripped and injected into the Low Energy Booster, the first of four synchrotrons that accelerate the protons to $20 \mathrm{TeV}$ [3].

To commission, tune, and monitor the SSCL linac, a set of diagnostics is being developed to measure the transverse and longitudinal characteristics of the beam [4]. The transverse set of diagnostics consists of the standard set of position monitors, wire scanners, and slit and collector emittance measurement units. The longitudinal diagnostics are the beam position monitors, energy absorber and collectors for low energy phase scans, and the Bunch Shapc Monitor.

The phase information from the beam position monitors gives longitudinal phase centroid information about the beam. The bunch shape is used to measure the longitudinal width of the beam in the phase or time dimension. The bunch shape monitor measurements separated by some known transformation can be used much like three wire scanner measurcments to reconstruct the longitudinal RMS emittance of the beam [5]. The first measurements of the beam's longitudinal characteristics have been made on the output beam from the $2.5 \mathrm{MeV}$ RFQ.

\footnotetext{
* Operated by the URA for the USDOE, under contract No. DE-AC35-89ER40486.
}

\section{DESCRIPTION}

Bunch Shape Monitors have been built and successfully used at the Moscow Meson Factory, [6], [7], [8], and at the FNAL linac [9], [10]. The basic concept and development history leading to the SSCL version of the Bunch Shape Monitor was reviewed at the 1992 Workshop on Beam Instrumentation in Berkeley [11].

Figure 1 illustrates the concept of the Bunch Shape Monitor. The $428-\mathrm{MHz}$ micro-bunches of the $\mathrm{H}^{-}$beam hit the

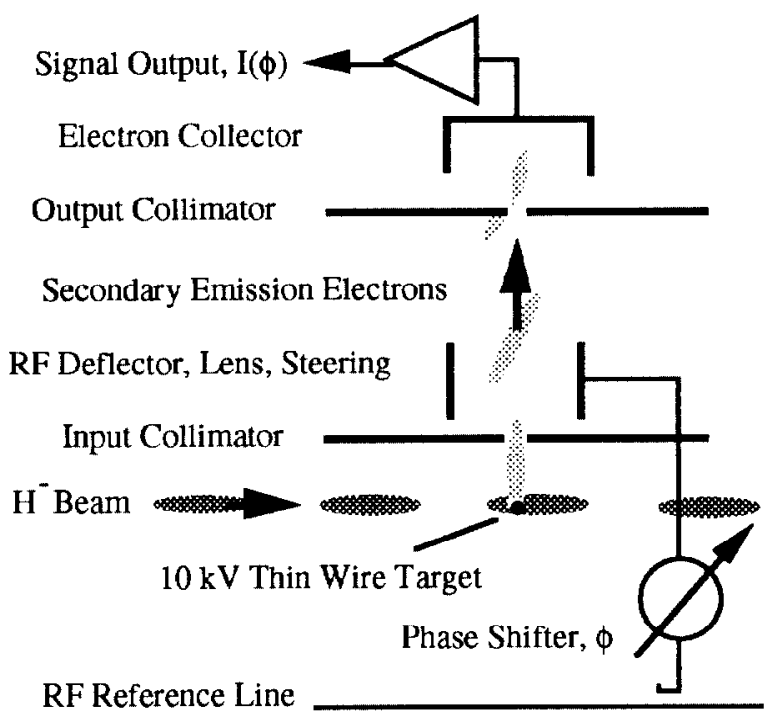

Figure 1. Functional diagram of Bunch Shape Monitor. Beam colliding with the target produces secondary emission electrons. The electrons have the same temporal structure as the beam. The rf deflector "time stamps" the secondary emission electrons which reach the electron collector.

target. The target is similar to a standard wire scanner with the wire at $10 \mathrm{kV}$. The $\mathrm{H}^{-}$beam hitting the target produces a proportional amount of secondary emission electrons. These electrons are created with averge energy of approximately $4 \mathrm{eV}$ and are quickly accelerated radially by the target potential. A well defined beam of electrons is created by the input collimator. The electron bunches have the same longitudinal structure as the $\mathrm{H}^{-}$beam. The beam passes into the if structure. The if deflector plates have a DC electrostatic field component which is used to focus the electron beam on the Output Collimator slit. The rf field sweeps the electron bunch across the Output Collimator slit. Only the thin slice 
of beam with the correct phase passes through the slit to the Electron Collector. The charge in this slice is proportional to the analogous slice of $\mathrm{H}^{-}$beam with the same relative phase. The Electron Collector is either a Faraday cup or an electron multiplier. The measured electron charge as a function of if phase gives the bunch shape of the $\mathrm{H}^{-}$beam. The resolution is calculated [12] to be approximately 7 picoseconds.

The new, compact design utilizes a quarter wave length parrellel wire line deflector combined with electrostatic focusing and steering electrodes. The Bunch Shape Monitor has been designed to fit in the nominal standard beam box for the SSCL linac. Bunch Shape Monitors are being developed for the RFQ-to-DTL matching section, DTL-to-CCL matching section, and the $600-\mathrm{MeV}$ Transport Line, as well as the Test model which will be used throughout the linac during commissioning.

\section{THEORETICAL RESULTS}

The first measurements of the bunch shape are on the output of the $428-\mathrm{MHz} \mathrm{RFQ}$. The RFQ accelerates the $\mathrm{H}^{-}$ beam to $2.5 \mathrm{MeV}$. The $\mathrm{H}^{-}$beam out of the RFQ has a nominal phase spread of 15 degrees and a longitudinal emittance of $0.13 \pi \mathrm{MeV}$-degree (RMS). The drift from the end of the RFQ to the Bunch Shape Monitor target is 148 $\mathrm{mm}$.

Theoretical results were obtained from simulations using PARMTEQ and PARMILA. Measured transverse emittances were used as the input beam. Longitudinal profiles were calculated at the bunch shape monitor. Earlier analysis had shown that image charges on the vanes did not contribute significantly for the SSCL RFQ. Therefore, the version of PARMTEQ we used had only space charge and multipole effects. Five thousand particles are used and the results are grouped in 3 degree wide bins. The calculated bunch shape at the nominal vane voltage setting is shown in figure 2 . The bunch shape was determined for all the particles in transverse phase space while the measured data is for a limited set as sampled by the target wire.

Bunch shapes were calculated for vane voltages from $80 \%$ to $120 \%$ of design vane voltages in steps of $5 \%$. The RMS width of the bunch shape and the change in phase center are calculated for each scan. The results are shown in figures 3 and 4 along with the measured data. To determine the design vane voltage, the transmission as a function of vane voltage was also calculated. The phase of the plot is relative to the synchronous particle.

\section{MEASUREMENT}

The beam bunch shape was measured for vane voltages from $80 \%$ to $112 \%$ of the nominal vane voltages in steps of $2.5 \%$ and $5 \%$. The nominal vane voltage was determined by measuring the knee of the transmission curve and by $x$-ray spectrum from electron traversing the RFQ gap. Figure 2

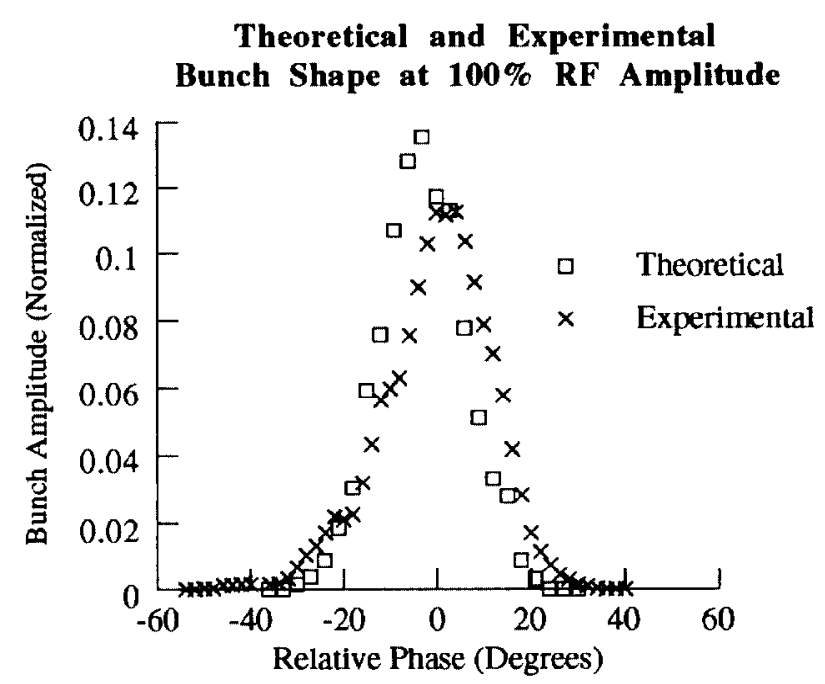

Figure 2. Theoretical bunch shape predicted by PARMTEQ simulation and experimental data as measured by the bunch shape monitor for nominal vane voltage. The intensity is normalized for a unit area.

shows the results of the measurement at the nominal vane voltage. The data is taken at a $1 \mathrm{MHz}$ sample rate during the beam pulse. The experimental data shown is an average over 10 sets of data during the middle of the beam pulse. The data is normalized for an area of one and shifted for an average phase of zero. Figures 3 and 4 show the measured rms width and centroid for various RFQ vane voltages. The phase is relative to the $R F$ drive line and is shifted from the cavity phase by an unknown constant. Therefore, to compare the measured and theoretical data, the phase of the experimental data is shifted such that the average is the same as the average of the theory.

\section{COMPARISON}

The RMS width and centroid compare well with the theory. Reproducibility at the $100 \%$ rf amplitude settings showed variations in the phase width and centroid of less than a few degrees. Error estimates have not been done for the simulations. The resolution of the measurement is expected to be approximately 1 degree. The sensitivity of the results to input phase space conditions for both the simulation and measurement have not been explored.

The actual bunch length measurement samples a thin line in transverse space. To get a meaningful number of particles in the results, the simulation uses all the particles across transverse space. Measurements must be performed across the beam width to determine the bunch shape transverse dependence. 


\section{CONCLUSION}

First results of measurements using the SSCL/TNR Bunch Shape Monitor are shown. The results are compared to theory. More work is required to quantify the accuracy and errors associated with the measurement. The Bunch Shape Monitor is also capable of measuring the micro-bunch shape as a function of time along the macro-bunch. These studies will be carried out at a later date. Additional bunch shape monitor systems are being developed for commissioning of

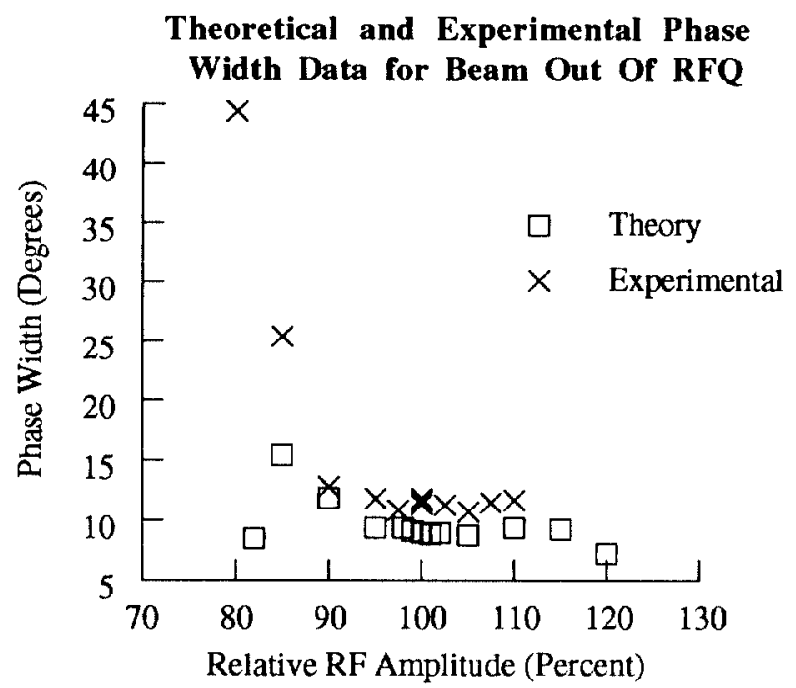

Figure 3. Measured and calculated RMS widths shown as a function of vane voltage. Widths are in degrees of $428 \mathrm{MHz}$.

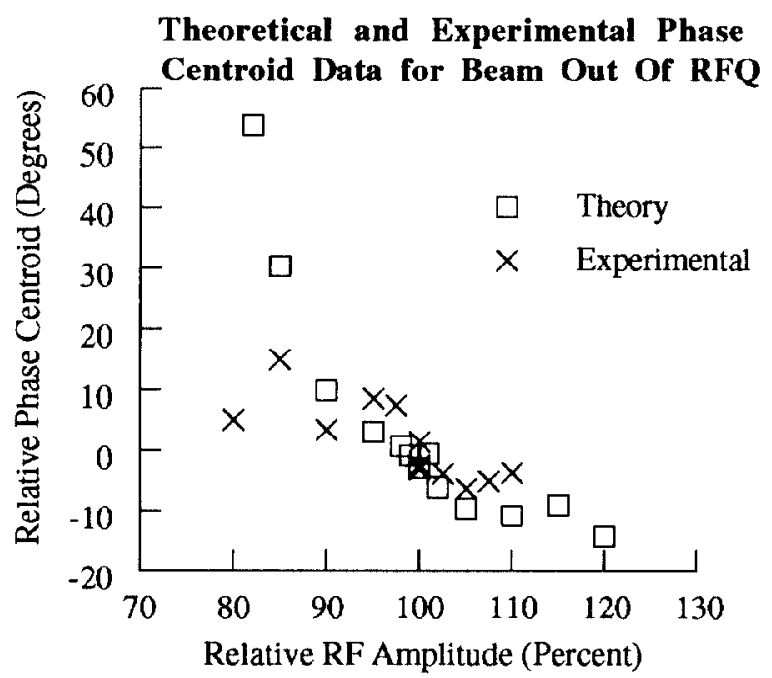

Figure 4. Measured and calculated micro-bunch centroids shown as a function of vane voltage. Widths are in degrees of $428 \mathrm{MHz}$. The centroid scale of fsets are arbitrary. The zero for the theoretical data is defined by the centroid for the nominal $(100 \%)$ vane voltage. The experimental data is shifted such that the average is the same as the theoretical data. the SSCL linac. They will be installed and tested in the matching sections into the DTL and CCL and at the end of the linac.

\section{ACKNOWLEDGMENTS}

Olin B. van Dyck of Los Alamos National Laboratory and Elliot S. McCrory of Fermi National Laboratory are acknowledged for their support and effort on the bunch shape monitor diagnostic.

\section{REFERENCES}

[1] A. V. Feschenko and P. N. Ostroumov, "Bunch Shape Measuring Technique and Its Application for an Ion Linac Tuning," Proceedings of 1986 Linac Conference, pp. 323-327.

[2] L. W. Funk, "The SSC Linac, 1992 Linear Accelerator Conference Proceedings," Vol. 1, pp. 8-12.

[3] Site-Specific Conceptual Design Report, SSCL Report SSCL-SR-1056, July 1990.

[4] J. W. Hurd et. al., "Beam Diagnostic Layout Requirements for SSCL Linac," these proceedings .

[5] Yu. V. Bylinsky et. al. "Longitudinal Emittance Measurement of the $100 \mathrm{MeV}$ Proton Beam," Conference Record of 1991 IEEE Particle Accelerator Conference, pp. 3062-3063

[6] Yu. V. Bylinsky et. al., "Initial Operation of the First $20 \mathrm{MeV}$ Tank of the INR Linac," Proceedings of the 1989 IEEE Particle Accelerator Conference, Vol. 3, pp. 1411-1413.

[7] G. I. Batskich et. al. "Proton Beam Acceleration Up to $160 \mathrm{MeV}$ at the Moscow Meson Factory Linac," Conference Record of the 1991 IEEE Particle Accelerator Conference, Vol. 5, pp. 3067-3069.

[8] V. A. Andreev et. al., "Development of RFQ Accelerator for the MMF Linac," Conference Record of the 1991 Particle Accelerator Conference, Vol. 5, pp. 3109-3110.

[9] E. McCrory et. al., "Use of an INR-Style Bunch-Length Detector," 1992 Linear Accelerator Conference Proceedings, Vol. 2, pp. 662-664.

[10] E. McCrory, private communication of data and results of preliminary beam measurements using FNAL bunch shape monitor, Feb. 2, 1993.

[11] A. V. Feschenko, "Bunch Shape Monitor Using Low Energy Secondary Electron Emission," proceedings of the Workshop on Beam Instrumentation, Berkeley, Oct. 1992.

[12] A. V. Feschenko and S. K. Esin, "Bunch Shape Monitor for the SSCL Linac, Preliminary Design Review Report," Academy of Science of Russia, Institute for Nuclear Research, Sept. 1992. 